\title{
REVUE INTERNATIONALE DE LA CROIX-ROUGE et \\ BULLETIN INTERNATIONAL des SOCIÉTÉS DE LA CROIX-ROUGE
}

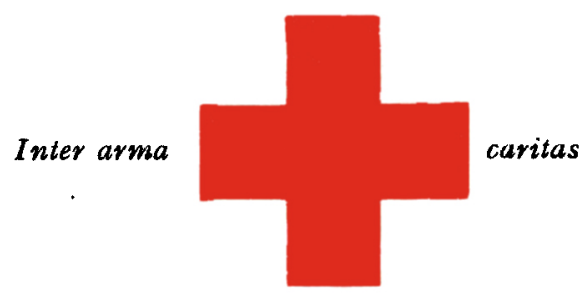




\title{
COMITE INTERNATIONAL DE LA CROIX-ROUGE
}

\section{à $G$ e n ève}

\author{
MAX HUBER \\ docteur en droit \\ ancien président de la Cour permanente \\ de justice internationale \\ president d'hosmeur \\ (1923) \\ PAUL RUEGGER \\ docteur en droit \\ ancien ministre de Suisse \\ en Italie et en Grande-Bretagne \\ president \\ (1948) 1
}

MARTIN BODMER

docteur en philosophie h. c. vice-prestaent

(1940)

\section{JACQUES CHENEVIERE}

docteur es lettres h. c.

(1919)

SUZANNE FERRIERE

directrice-adjointe de l'International Social Service

(1924)

\section{GEORGES PATRY}

docteur en médecine

colonel, ancien médecin de division de l'armée suisse

$$
\text { (1929) }
$$

\section{LUCIE ODIER}

ex-chef du Service des infirmières-visiteuses de la Croix-Rouge genevoise

(1930)

\section{CARL J. BURCKHARDT}

docteur en philosophie

ancien ministre de Suisse en France

(1033)

\section{EDOUARD CHAPUISAT}

docteur ès lettres $\mathbf{h}$. c. (1938)

ALEC CRAMER

docteur en médecine, colonel

$$
\text { (1938) }
$$

ADOLF-L. VISCHER

docteur en médecine

$$
\text { (1945) }
$$

\section{LÉOPOLD BOISSIER}

docteur en droit

professeur à l'Université de Genève

secrétaire général de l'Union interparlementaire

$$
\text { (1946) }
$$

\author{
ERNEST GLOOR \\ docteur en médecine \\ vice-president \\ (1945)
}

RENÉ VAN BERCHEM

docteur en droit, banquier

(1946)

\author{
PAUL CARRY \\ docteur en droit \\ professeur à l'Université de Genève \\ (1946) \\ EDMOND GRASSET \\ docteur en médecine \\ professeur à l'Université de Genève \\ directeur de l'Institut d'hygiène \\ (1946)
}

\section{CLAUDE DU PASQUIER}

docteur en droit colont! divisiontlaire professeur aux Universités de Genève et Neuchatel

(1947)

HENRI GUISAN

général

ancien commandant en chef de l'armée sujsse

(1948)

ALFREDO VANNOTTI

docteur en médecine professeur à I'Université de Lausanne (1949)

\section{RODOLFO OLGIATI}

ancien directeur du Don suisse (1949)

1 Les années indiquées dans les parenthèses désigneut les dates de nomination des membres du Comité international. 


\title{
REVUE INTERNATIONALE DE LA CROIX-ROUGE TRENTE ET UNIEME ANNEEE

\section{SOMMAIRE}

\begin{abstract}
COMITE INTERNATIONAL DE LA CROIXROUGE. - Jean S. Pictet, directeur-délégué du Comité international. Les nouvelles Conventions de Genève. La rétention du personnel sanitaire des armées tombé au pouvoir de la partie adverse (suite et fin), 937. - Le Comité international de la Croix-Rouge et l'aide aux réfugiés de Palestine (H.C.) (hors-texte), 977. - Discours prononcé le 25 novembre 1949 à Lake Success par M. P. Ruegger, président du Comité international de la Croix-Rouge, devant la Commission politique spéciale de l'Assemblée des Nations Unies, 989. - Activité du Comité international de la Croix-Rouge en faveur de la population civile allemande, 996 .
\end{abstract}

A TRAVERS LES REVUES, rooo.

\section{BULLETIN INTERNATIONAL DES SOCIÉTES DE LA CROIX-ROUGE, Ioo3.}

COMITÉ INTERNATIONAL. - LIGUE DES SOCIÉTÉS DE LA CROIX-ROUGE. - NOUVELLES DES SOCIÉTÉS DE LA CROIX-ROUGE, DU CROISSANT-ROUGE ET DU LION ET SOLEIL ROUGES.

\author{
La Revue internationale de la Croix-Rouge \\ ef \\ Bultetin des Societes de la Croix-Rouge \\ parait à la fin de chaque mois
}

\begin{abstract}
Eprrzur: Comité international de la Croix-Rouge - Repacteur responsable: Louis Demolis Répaction: Louis Demolis, Jean-G. Lossier, membres du Secrétariat du Comité international de la. Croix-Rouge - Administration : Louis Demolis.
\end{abstract}

Prix, franco un an : Fr. 20,- . Le numéro: Fr. 2,-. Abonnements, vente au numéro, publicité, au Siège du Comité international 7, avenue de la Paix, Genève, Téléphone : 20560 , Compte de chèques I 1707 
SUPPLEMENTS DE LA REVUE INTERNATIONALE DE LA CROIX-ROUGE

SOMMAIRE DU SUPPLEMMENT EN LANGUE ANGLAISE, DECEMBER 1949, No I2, VOL. II:

\section{Contents}

Seventy-fifth birthday of M. Max Huber.

A tribute to M. Jacques Chenevière, Member of the International Committee of the Red Cross.

Jean S. Pictet, Director-Delegate of the Committee of the Red Cross. The New Geneva Conventions: Retention of members of the Army Medical Services who have fallen into the hands of the Enemy.

ICRC Relief to the German Civil Population.

Table of Contents. Vol. II (I949).

SOMMAIRE DU SUPPLEMENT EN LANGUE ESPAGNOLE, DICIEMBRE I949, No I2, VOL.I.

\section{SUMARIO}

El 75. Cumpleaños del Sr. Max Huber.

Homenaje al Sr. Jacques Chenevière, miembre del Comité Internacional de la Cruz Roja. Un Jubileo.

Actividades del Sr. Jacques Chenevière en el seno del Comité Internacional de la Cruz Roja.

Jean S. Pictet, director delegado del Comité Internacional de la Cruz Roja. Los nuevos Convenios de Ginebra : La retención del personal sanitario de los ejércitos caĺdo en poder del Enemigo.

Indice de Sumarios. Vol. I (I949). 


\section{Votre Banque...}

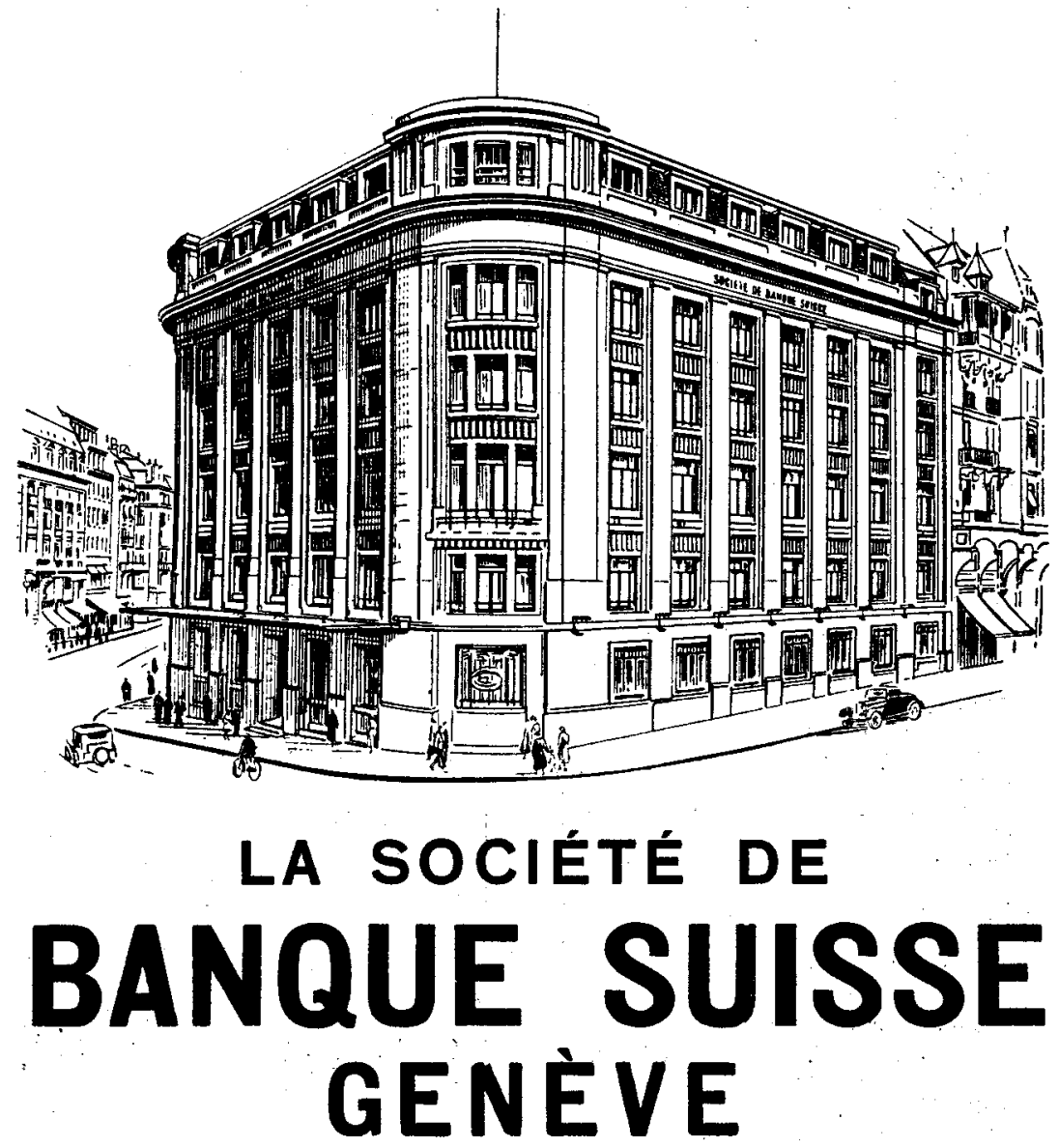

2, rue de la Confederation

BALE ZURIOH ST-GALL LAUSANNE NEUCHATEL OHAUX-DE-FONDS SOHAFFHOUSE BIENNE LONDRES NEW-YORK

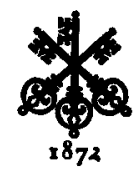

CAPITAL ET RÉSERVES FRS 200 MILLIONS 


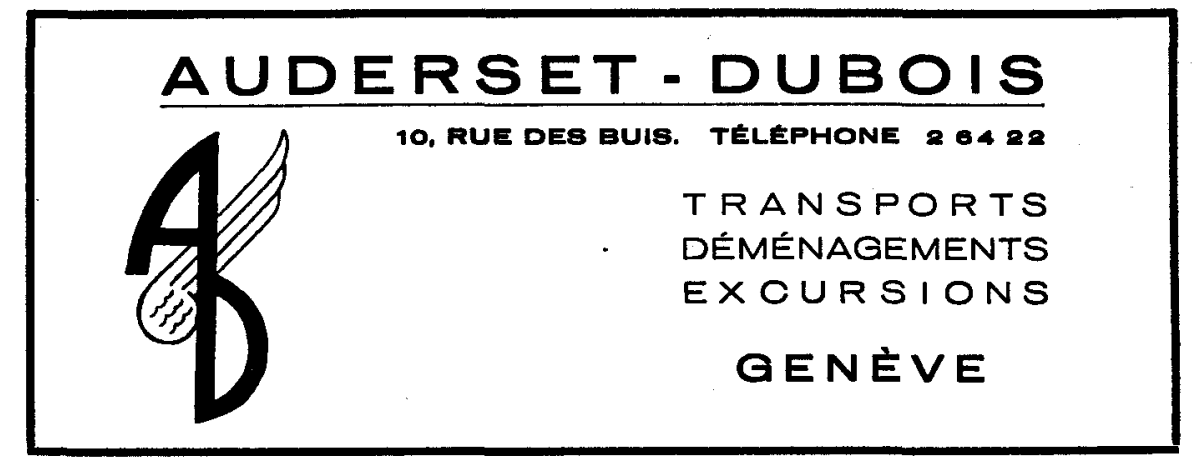

\section{HEBERLÉ S.A.}

\section{FOURNITURES INDUSTRIELLES} EN GROS

\section{GENEVE}

Rue du Rhóne 61 et Grand-Qual 42 Tolephone 41377 (2 lignes)

\section{Amiante}

Bottes en caoutchouc

Chiffons d'essuyages blancs et couleurs

Courroies de transmissions caoutchouc, cuir ou tissées

Déchets de coton d'essuyage blancs et couleurs

Eponges

Feutre industriel

Joints en tous genres

Peaux de daim

Produits abrasifs $*$ SIA ,

Tabliers industriels en caoutchouc, cuir, GURIT, toile

Tapis en caoutchouc

Tuyaux d'arrosage, à air comprimé, à vin, à vapeur, pour radiateurs

Tous tuyaux caoutchouc pour l'industrie



ENTREPRISE GÉNÉRALE

\section{ÉLECTRICITÉ ET TÉLÉPHONE}

M. FORNASARI

5, avenue Henri-Dunant 


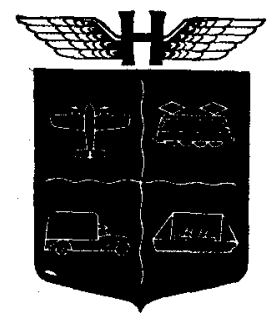

Compagnie d'Assurances Générales

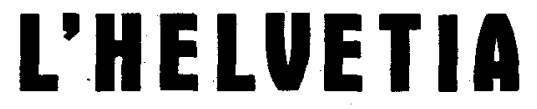

\section{ST-GALL (Suisse)}

Fond be en 1858

\section{ASSURANGES TRAMSPORTS}

et

REASSURAHCES dans toutes les Branchos

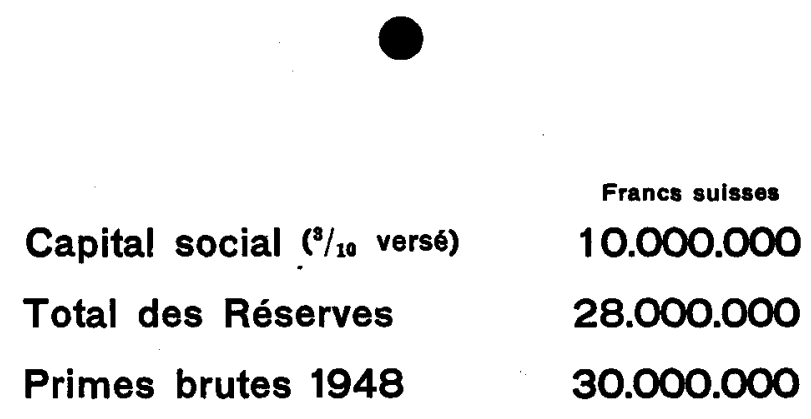






Boîtes

métalliques

en tous genres

Emballages

do carlonnage

Boltes * METALLO -

\section{JHIO IFIFIM AI N N IEJRJEIRTES THOUNE}

\section{PAPETERIE DE BALSTHAL}

baLsthal (Solouro)

OUATE DE CELLULOSE

PAPIER CRÊPÉ POUR PANSEMENTS

LINGERIE EN PAPIER

Bureau de vente:

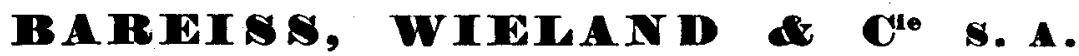
Kornhausbrücke, 7 Z ZURICH - Téléphone 234724 

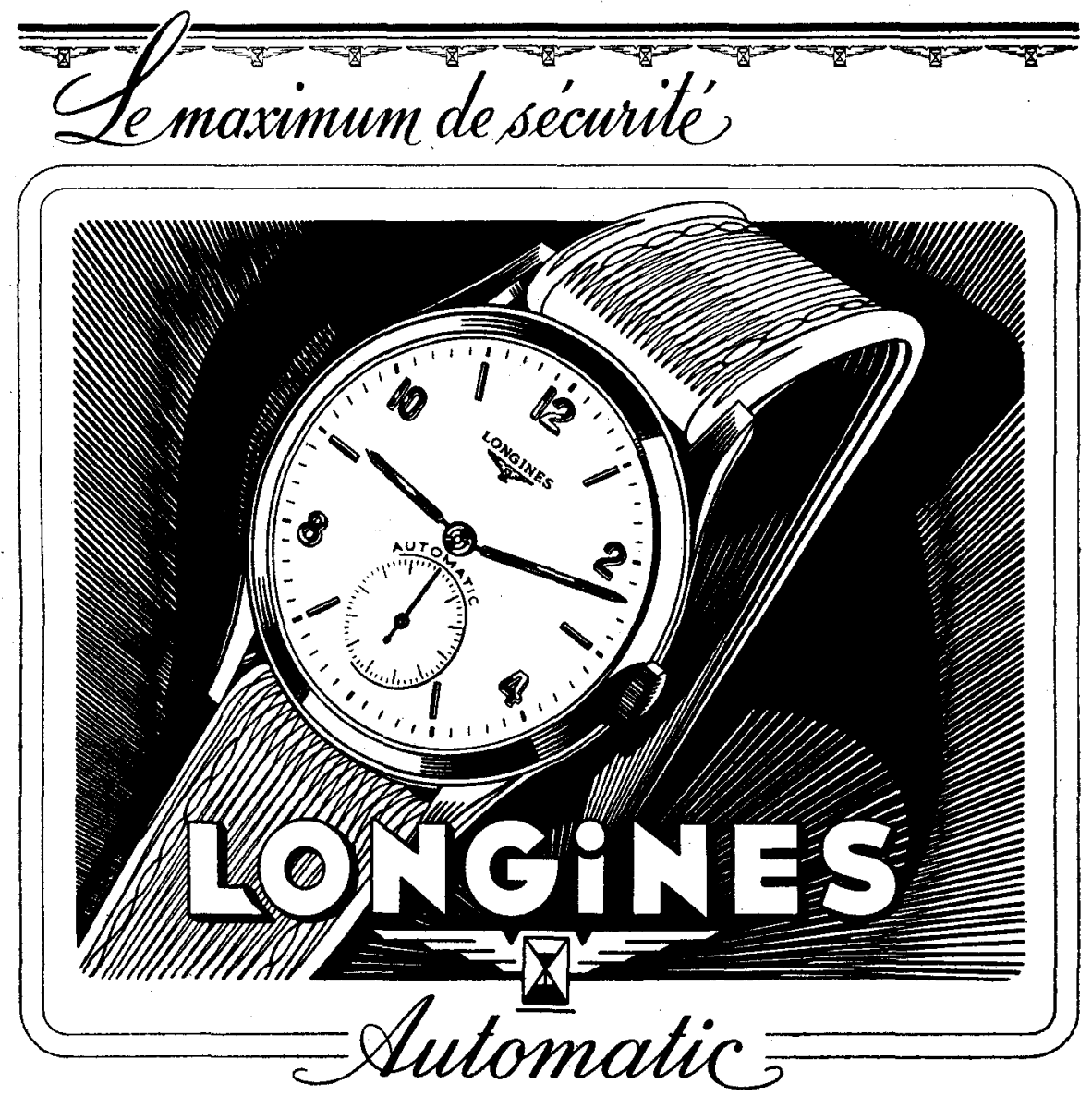

La montre automatique Longines se remonte d'elle-même, silencieusement, en moins de 3 heures et sa réserve de marche dépasse $3^{6}$ heures. Equipée d'un double système de protection contre les chocs, elle est, de plus, dotée d'une stabilité de réglage remarquable.

Antimagnétique, protégée contre la poussière et l'humidité, "Longines Automatic» illustre les qualités "Longines" jusque dans ses moindres détails.

LA COMPAGNIE DES MONTRES LONGINES ET SES AGENTS DANS LE MONDE ENTIER VOUS ASSURENT LONGINES LA GARANTIE DU SERVICE MONDIAL LONGINES.

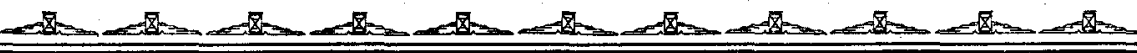



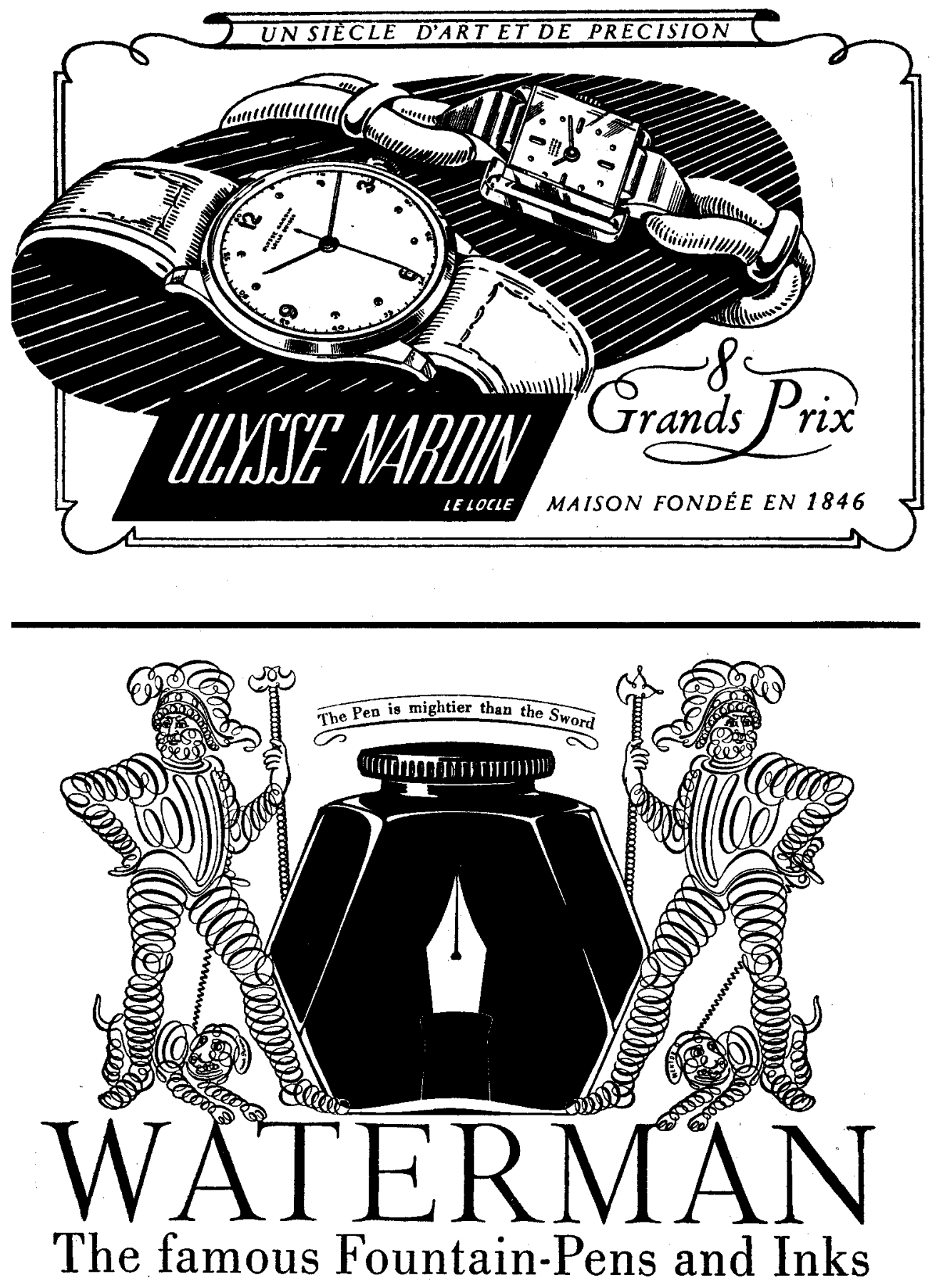


\section{SOCIÉTÉ ANONYME FIDUGIAIRE SUISSE}

(Schweizerische Treuhandgesellschaft)

Fondée en 1906

\section{BALE}

ZURICH - GENEVE - LAUSANNE

CONTRÔLE OBLIGATOIRE DE BILANS

selon art. 723, 727, 732, et 906 C.O.

\section{QUESTIONS FISOALES}

Fonctions de fidéi-commissaires, liquidations, expertises, arbitrages

\section{LA BALOISE}

COMPAGNIE D'ASAURANCES contre les risques de transport

BALE (Suisse)

\section{Fondée en 1864}

La maison de confiance qui est spécialisée pour les assurances maritimes, fluviales, terrestres et aériennes. 


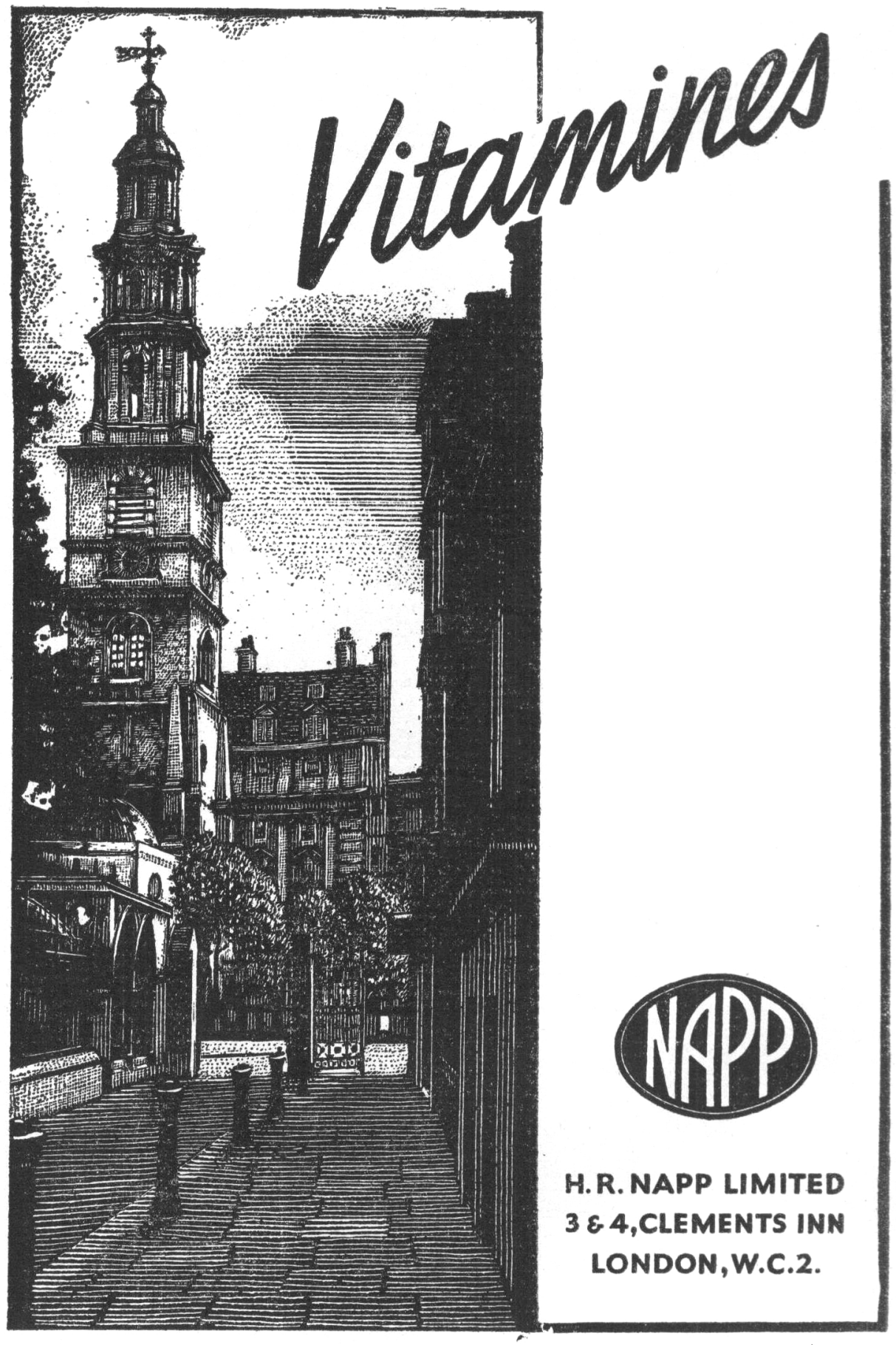

